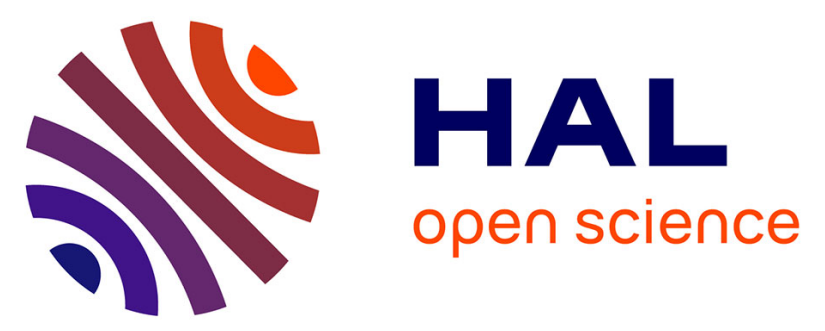

\title{
Analytical Model for External Induction Variations of a Ferromagnetic Cylinder Undergoing High Mechanical Stresses in a Low Magnetic Field of any Orientation
} Antoine Viana, Laure-Line Rouve, Gilles Cauffet, Jean-Louis Coulomb

\section{- To cite this version:}

Antoine Viana, Laure-Line Rouve, Gilles Cauffet, Jean-Louis Coulomb. Analytical Model for External Induction Variations of a Ferromagnetic Cylinder Undergoing High Mechanical Stresses in a Low Magnetic Field of any Orientation. IEEE Transactions on Magnetics, 2011, 47 (5), pp.1366-1369. hal-00596840

\author{
HAL Id: hal-00596840 \\ https://hal.science/hal-00596840
}

Submitted on 30 May 2011

HAL is a multi-disciplinary open access archive for the deposit and dissemination of scientific research documents, whether they are published or not. The documents may come from teaching and research institutions in France or abroad, or from public or private research centers.
L'archive ouverte pluridisciplinaire HAL, est destinée au dépôt et à la diffusion de documents scientifiques de niveau recherche, publiés ou non, émanant des établissements d'enseignement et de recherche français ou étrangers, des laboratoires publics ou privés. 


\title{
Analytical Model for External Induction Variations of a Ferromagnetic Cylinder Undergoing High Mechanical Stresses in a Low Magnetic Field of Any Orientation
}

\author{
Antoine Viana, Laure-Line Rouve, Gilles Cauffet, and Jean-Louis Coulomb \\ Grenoble Electrical Engineering Lab, Université de Grenoble, (Grenoble-INP, UJF, CNRS UMR 5269), \\ BP 46, 38402 Saint Martin d'Hères, France
}

\begin{abstract}
This paper introduces an original approach for characterizing and modeling the external induction variation of a complex ferromagnetic structure undergoing magneto-mechanical effects. From the Jiles Law of Approach that describes the intrinsic magnetization changes due to magnetostriction, an expression of the law in terms of conveniently external measurable induction $B$ is derived for thin ferromagnetic devices. Based on measurements of induction performed by external sensors on an internally pressurized cylinder, an analytical model is found. This model is then tested for any orientation of the magnetic field with respect to stress, from parallel to orthogonal.
\end{abstract}

Index Terms-Analytical, Jiles, low field, magneto-mechanical.

\section{INTRODUCTION}

$\mathbf{O}$ UR AIM is to anticipate the magnetic induction variation measured on magnetic sensors located outside a ferromagnetic material, when the latter is undergoing high mechanical stresses in a low magnetic field. Several macroscopic models for magneto-elastic effects can be found in literature. The macroscopic model developed by Jiles and Atherton [1] seems particularly well suited to our approach. This model clearly stems that under stress, magnetization tends towards the anhysteretic magnetization. Jiles and Atherton have based part of these observations from experiments done in the past by various authors, including members of our laboratory [2].

The Jiles model describes scalar variations of magnetization M. For this reason, to test the model, most studies generally deal with simple shapes, free of demagnetizing field effects, such as rods. In these cases, as $\mathbf{M}$ is quite homogeneous inside the material, external induction measurement gives direct characterization of the internal magnetization. Moreover, for these shapes, magnetization is also collinear to internal stress.

Our prototype is a thin and hollow ferromagnetic cylinder, internally pressurized. For such geometry, exhibiting an important form effect, magnetization $\mathbf{M}$ is no more homogeneous and cannot be simply extracted from external induction B. Nevertheless, the measurements we performed on our prototype demonstrated that the phenomenological behavior depicted by Jiles and Atherton for the intrinsic magnetization $\mathbf{M}$ was also verified by induction $\mathbf{B}$ outside the material [3]: external tri-axial magnetic sensors, located around our prototype, showed that induction $\mathbf{B}$, while mechanical stress was raised, was converging on the anhysteretic curve. The theoretical justification for that can be found in [4]: for thin ferromagnetic shells, the distribution of magnetization $\mathbf{M}$ in the material can be linked to induction $\mathbf{B}$ measured at a point $P$ outside the

Manuscript received May 31, 2010; accepted September 11, 2010. Date of current version April 22, 2011. Corresponding author: A. Viana (e-mail: antoine. viana@g2elab.grenoble-inp.fr).

Color versions of one or more of the figures in this paper are available online at http://ieeexplore.ieee.org.

Digital Object Identifier 10.1109/TMAG.2010.2078801 material, by a matrix depending only on $P$-coordinates, and on the geometry of the shell. Thus, external induction $\mathbf{B}$ appears to be a spatial average of magnetization distribution on the surface cylinder.

In a previous study [3], a simple analytical expression of the Jiles equation was derived for external induction $\mathbf{B}$ when the inductor field was vertical: for this configuration, stress, and magnetization were parallel inside the material, which correspond to a scalar magnetostriction model.

In this paper, external $\mathbf{B}$ variations are measured when the inductor field is parallel to the cylinder axis. In this case, magnetization and stress are no more collinear but orthogonal inside the material. A more complex and general analytical law is then exhibited. Finally, an analytical vectorial law is proposed for external $\mathbf{B}$ variation due to stress. It is valid for any direction of inductor field and compared with measurements.

\section{JILES-ATHERTON LAW OF APPROACH}

Jiles and Atherton [1] postulated a scalar law describing the behavior of magnetization $\mathbf{M}$ for a ferromagnetic material undergoing mechanical stresses $\sigma$. This law originally separates magnetization in a reversible contribution $\mathbf{M}_{\mathbf{R E V}}$, and an irreversible contribution $\mathbf{M}_{\text {IRR }}$, respectively, expressed as

$$
\begin{aligned}
\frac{d M_{\mathrm{REV}}}{d W} & =c\left(\frac{d M_{\mathrm{REV}}}{d W}-\frac{d M_{\mathrm{IRR}}}{d W}\right) \\
\frac{d M_{\mathrm{IRR}}}{d W} & =\frac{1}{\xi}\left(M_{\mathrm{ANH}}-M_{\mathrm{IRR}}\right)
\end{aligned}
$$

$W=\sigma^{2} / 2 E$ denotes the mechanical energy density $\left(\mathrm{J} / \mathrm{m}^{3}\right), \xi$ is a coefficient with dimension of energy density $\left(\mathrm{J} / \mathrm{m}^{3}\right)$, which accounts for the rate of change of magnetization with stress, $E$ is the Young modulus $(\mathrm{Pa}), c$ a coefficient of dimension of unit, describing the flexibility of the domain walls, $M_{\mathrm{ANH}}$ the anhysteretic magnetization $(\mathrm{A} / \mathrm{m})$, function of stress.

Given that

$$
M=M_{\mathrm{REV}}+M_{\mathrm{IRR}} \text {. }
$$

The variation of magnetization $M$ can then be expressed as

$$
\frac{d M}{d \sigma}=\frac{\sigma}{\xi E}\left(M_{\mathrm{ANH}}-M\right)+c \frac{d M_{\mathrm{ANH}}}{d \sigma} .
$$




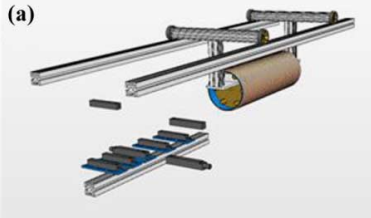

(b)

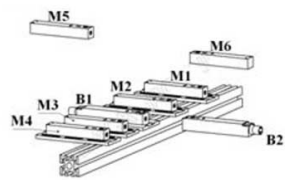

(c)

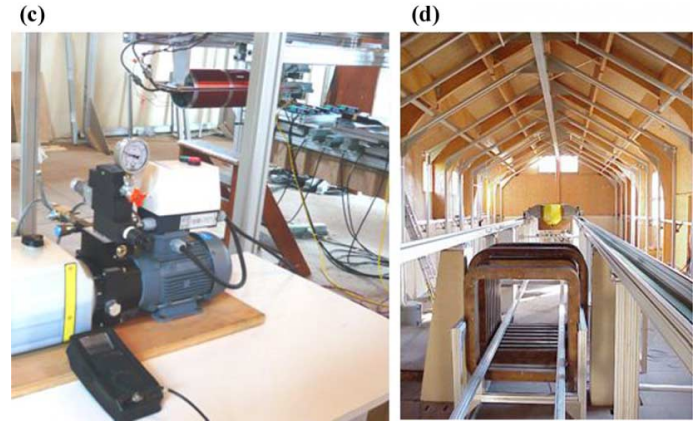

Fig. 1. (a), (b) Location of the sensors. (c) The hydraulic pump, and the cylinder inserted into a longitudinal solenoid used for demagnetization or polarization. (d) The LMMCF measurements building is oriented in the N-S direction. The coils used for controlling the ambient field are visible, as well as the rails used for signature measurements.

In (4), some questions can occur regarding the conditions under which derivative of $M$ with regards to stress must be calculated. Equation (4) is given as the variation of $\mathbf{M}$ in a given magnetic field $\mathbf{H}$. Field $\mathbf{H}$ must be understood as the applied magnetic field, not the internal field. In a ferromagnetic body, the internal field is the sum of the applied field $\mathbf{H}_{\mathbf{0}}$ and the demagnetizing field $\mathbf{H}_{\mathbf{m}}$ due to the material. The latter, at a point $P$, depends upon magnetization $\mathbf{M}$ through the shape factor $N: \mathbf{H}_{\mathbf{m}}(P)=-N(P) \cdot \mathbf{M}(P)$. This shape factor is only a function of point $P$ and of the geometry of the sample, not of any physical property of the material: changes of magnetization with stress produce the variation of the demagnetizing field. Thus, derivative of magnetization $\mathbf{M}$ with stress is implied at fixed applied magnetic field $\mathbf{H}_{\mathbf{0}}$

$$
\left.\frac{\partial \boldsymbol{M}}{\partial \sigma}\right|_{\boldsymbol{H}_{\mathbf{0}}}=\frac{\sigma}{\xi E}\left(\boldsymbol{M}_{\boldsymbol{A N H}}-\boldsymbol{M}\right)+\left.c \frac{\partial \boldsymbol{M}_{\boldsymbol{A N H}}}{\partial \sigma}\right|_{\boldsymbol{H}_{\mathbf{0}}} .
$$

Confusion about the meaning of $\mathbf{H}$ may arise because the usual mockups studied in previous papers exhibited geometries for which the demagnetizing field was negligible. For simple geometries such as a rod, undergoing an external magnetic field parallel to its longitudinal axis, the internal field is close to the applied field.

Equation (5) has no general analytical solution, except when the anhysteretic magnetization is independent from stress $\sigma$. In addition, to be numerically solved, it is necessary to know the following:

a) values for coefficients $\mathrm{c}$ and $\xi$ ( $\mathrm{E}$ is usually known);

b) the law of evolution of the anhysteretic magnetization with stress $\mathbf{M}_{\mathbf{A N H}}(\sigma)$ under the constant applied field $\mathbf{H}_{\mathbf{0}}$. As shown in [3], (5) can be written by using external $\mathbf{B}$ instead of internal $\mathbf{M}$.

No general solution exists for this equation. In the following parts, external B measurements are made to characterize its behavior with stress. Then an analytical solution is proposed and tested.

\section{EXPERIMENTAL SETUP AND MEASUREMENTS}

\section{A. LMMCF Facility}

The Laboratory of Magnetic Metrology in Weak Fields (LMMCF) is located in Grenoble, France [see Fig. 1(d)]. In this experimental facility, research and measurements are conducted in the area of very low magnetic fields (around a few nT signatures). A magnetic environment simulator has been designed and built to compensate local earth field and to create any field between $\pm 70000 \mathrm{nT}$, with an homogeneity close to $10^{-3}$ over a large volume $(27 \times 2 \times 2 \mathrm{~m})$. The system is based on a tri-axial set of coils, driven by bipolar generators. Mock-ups can be mounted on a trolley driven over a pair of rails by an electrical motor along the longitudinal direction, allowing magnetic signature measurements in a $[-1.5 \mathrm{~m}, 1.5 \mathrm{~m}]$ range above fixed magnetic sensors [see Fig. 1(d)].

\section{B. Description of the Prototype}

The prototype is a ferromagnetic hollow cylinder of axis $\mathbf{Z}$. Dimensions are length $L=0.5 \mathrm{~m}$, internal radius $R \gg e, e$ being the thickness of the cylinder. The latter, filled with hydraulic oil, is subjected to an internal pressure up to 100 bars, driven by an external pump [see Fig. 1(c)]. A pressure sensor mounted inside the cylinder allows an accurate measurement of internal pressure.

The system has been designed in order to let both ends of the cylinder free in displacement when pressure increases. Thus, the mechanical approach was to work in plane stresses. As a consequence, the $\sigma_{\mathrm{zz}}$ stress tensor component is null. The main stress is $\sigma_{\theta \theta}$. In addition, stresses are proportional to internal pressure. Thus, plots representing magnetization versus stress are identical to those representing magnetization versus internal pressure.

Eight tri-axes Fluxgate sensors are used (denoted M1 to M4 and B1 and B2) with a dynamic range $+/-100 \mu \mathrm{T}$, a bandwidth from DC to $1 \mathrm{KHz}$, a sensitivity of $1 \mathrm{nT}$ [see Fig. 1(b)]. The set of eight sensors allows the following two kinds of measurements.

- Signature Measurement (Fig. 2): In this case, the cylinder is moving along the rails and signatures are performed. Given the location of the sensors, five signatures are duplicated.

- Static Measurement During an HPP Cycle (Fig. 3): In this case, the cylinder is static, centered above sensor B1. Variation of induction versus pressure is monitored on the eight sensors.

\section{Magnetic Characterization of the Cylinder}

1) Anhysteretic Signature Measurements: A magnetic characterization of the cylinder was first achieved, for the determination of the anhysteretic inductions under several static pressures.

For anhysteretic induction measurements under pressure P0 and field B0, the cylinder was first zero-stress demagnetized, and pressure was then raised to $\mathrm{P} 0$. The cylinder was then subjected to a large decaying sinusoidal field, in the presence of the polarization induction B0. Then, a signature was done while the pressured cylinder was shifted above the sensors. This operation was repeated for several increasing values of B0, and for several pressure values in 0-100 bar range. Measurements showed that the anhysteretic induction was independent from pressure for a vertical polarization field [see Fig. 4(a)], whereas 

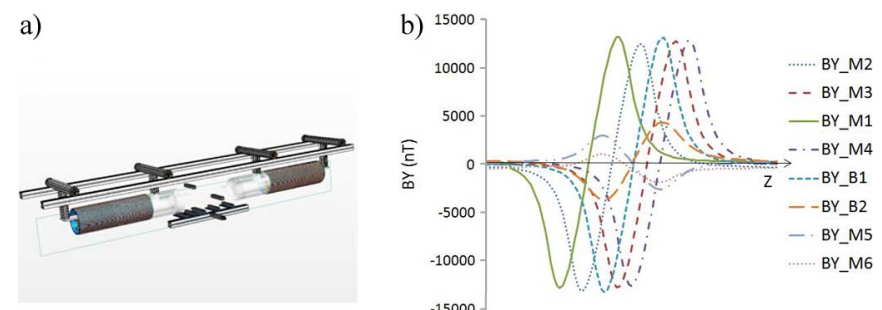

Fig. 2. Induction B signature (a) the cylinder is translated over the eight sensors. (b) Typical magnetic signature curve obtained for each of the three components of induction B. The plot represents the vertical component BY of induction $\mathrm{B}$ under a longitudinal inductor field $B 0 L=40 \mu \mathrm{T}$. The plot is characteristic of the orthogonal component $Y$ of a $Z$ dipole signature. a)

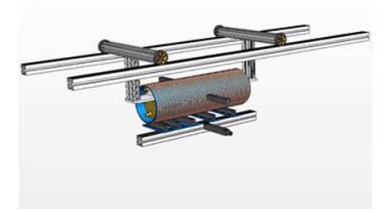

Fig. 3. External induction change measurement under a constant vertical inductor field B0V, while pressure is first raised, then released. (a) The cylinder is static, centered above B1 sensor. (b) Vertical component BY_B1 of induction measured on centered sensor B1, during a HPP cycle under a vertical inductor field $B 0 \mathrm{~V}=40 \mu \mathrm{T}$.

the anhysteretic induction under a longitudinal field was a linear function of pressure [see Fig. 4(b)].

2) HPP Cycles Measurements: The second series of measurements was focused on the variation of external induction $\mathbf{B}$, while pressure was raised, then relaxed, under a constant applied induction $\mathbf{B}_{\mathbf{0}}$ of any direction. Experimentally, such HPP cycle measurement consists of three steps. First, a particular initial magnetic state is conferred on the cylinder: zero-stress demagnetization, or anhysteretic polarization under a given applied external induction $\mathbf{B}_{\mathbf{P O L}}$. Second, the inductor field is set to B0. Finally, pressure is raised up to $100 \mathrm{bars}$, and then relaxed to 0 bar. A magnetic signature can be performed under a static pressure, during the HPP cycle. In this case, the cylinder, initially static [see Fig. 3(a)], is translated along the sensors [see Fig. 2(a)] while pressure $P_{\mathrm{MES}}$ is maintained. Once the signature is performed, the cylinder is sent back to its original location, and the end of the HPP cycle (pressure release) is recorded.

Several HPP cycles series were done with different initial states and inductor fields (see Fig. 4). Remember that when the applied field is parallel to the cylinder axis (i.e., longitudinal), magnetization and stress are orthogonal inside the material.

In all cases, induction converges towards its anhysteretic value under pressure, independently from the initial polarization configuration. If the vertical anhysteretic induction does not depend on stress [see Fig. 4(a)], the longitudinal one presents a quite linear variation with pressure [see Fig. 4(b)]. This may mostly be the case for intrinsic magnetization: because of the more light shape effect, permeability variations caused by any constraints (magnetostriction, temperature,...) affects more longitudinal magnetization than vertical one, inside a hollow cylinder. Initially, for both vertical and longitudinal orientations of inductor field, external induction presents an exponential variation.

In addition, measurements showed that the measured induction during an HPP cycle was linear with regards to the inductor field. Consequently, induction changes observed on each

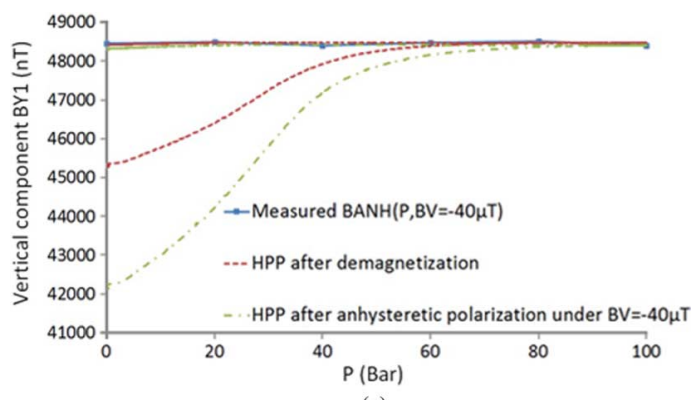

(a)

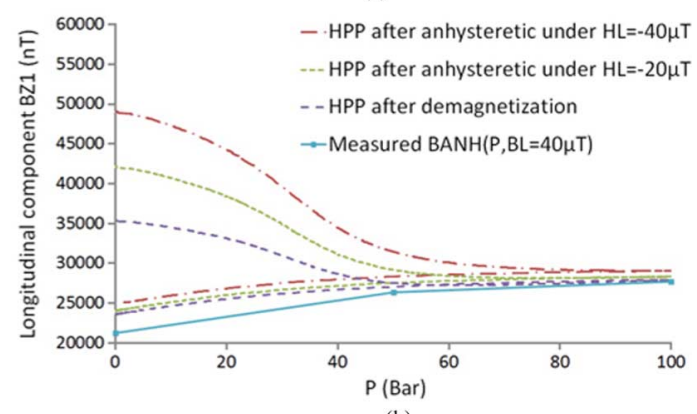

(b)

Fig. 4. (a) (respectively, b): Variation of the vertical (respectively, longitudinal) component BY1 (respectively, BZ1) of induction B during an HPP cycle, measured on central sensor B1. Inductor field is $B 0 \mathrm{~V}=40 \mu \mathrm{T}$ (respectively, $B 0 L=40 \mu \mathrm{T})$. For all cycles, induction migrates towards its anhysteretic value, independently from the initial magnetic state of the cylinder. analytical model for induction $\mathbf{B}$.

sensor during an HPP cycle performed under any inductor field $\mathbf{B 0}=\lambda \mathbf{B O L}+\mu \mathbf{B O V}$ can be deduced from those measured separately using two HPP cycles, operated under the two reference inductor fields BOL then BOV

$$
\boldsymbol{B}(P, \boldsymbol{B 0})=\lambda \boldsymbol{B}(P, \boldsymbol{B O} \boldsymbol{L})+\mu \boldsymbol{B}(P, \boldsymbol{B O} \boldsymbol{V}) .
$$

The experienced linearity of $\mathbf{B}$ is in agreement with the expected linear behavior of induction in the Rayleigh low fields region.

Inside a thin ferromagnetic shell, the strong form effect prevents any magnetization perpendicular to the surface to arise, and magnetization remains tangential to the shell. In that particular case, it was shown [3] that the Jiles-Atherton model for magnetization changes could be expressed in terms of induction B

$$
\left.\frac{\partial \boldsymbol{B}}{\partial \sigma}\right|_{\boldsymbol{B}_{\mathbf{0}}}=\frac{\sigma}{\xi E}\left(\boldsymbol{B}_{A N H}-\boldsymbol{B}\right)+\left.c \frac{\partial \boldsymbol{B}_{\boldsymbol{A} \boldsymbol{N} \boldsymbol{H}}}{\partial \sigma}\right|_{\boldsymbol{B}_{\mathbf{0}}} .
$$

\section{Analytical Model for a Vertical Inductor Field}

Given the physical symmetry of the problem, when the cylinder is subjected to a vertical inductor field, magnetization $\mathbf{M}$ in the shell cannot have a longitudinal component MZ. In addition, measurements showed that for a vertical polarization field $\mathbf{B O V}$, the transverse and vertical components $B_{\mathrm{ANH}} \mathrm{Xi}$ and $B_{\mathrm{ANH}} \mathrm{Yi}$ of the anhysteretic induction $\mathbf{B}_{\mathbf{A N H}}$ measured on any sensor Si were independent from stress [see Fig. 4(a)]. Consequently, the second term of (7) collapses and an analytical solution for the variation of $\mathrm{BXi}$ and $\mathrm{BYi}$ with stress, for a given sensor $\mathrm{Si}$, can be found

$$
\begin{aligned}
B X i(P, \boldsymbol{B O} \boldsymbol{V}) & =B_{A N H} X i(\boldsymbol{B O V}) \\
+ & {\left[B_{0} X i-B_{A N H} X i(\boldsymbol{B} \mathbf{V})\right] \times e^{-P^{2} / 2 E \xi} } \\
B Y i(P, \boldsymbol{B O V}) & =B_{A N H} Y i(\boldsymbol{B O} \boldsymbol{V}) \\
+ & {\left[B_{0} Y i-B_{A N H} Y i(\boldsymbol{B O V})\right] \times e^{-P^{2} / 2 E \xi} }
\end{aligned}
$$




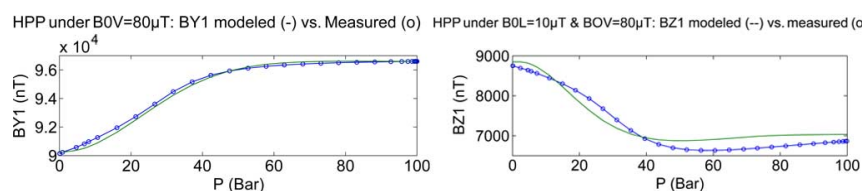

Fig. 5. Left: modeled (-) versus measured (o) variation of BY1 during an HPP cycle under $B 0 \mathrm{~V}=80 \mu \mathrm{T}$. This HPP cycle yields determination of $\xi$. Right: Modeled (-) versus measured (o) variation of BZ1 during an HPP cycle under $B 0 L=10 \mu \mathrm{T}+\mathrm{BOV}=80 \mu \mathrm{T}$. On centered sensor $\mathrm{B} 1, \mathrm{BY} 1$ is close to zero.

$P$ is the pressure in bar, $\mathrm{B}_{0} \mathrm{Xi}$ (respectively, $B_{0} \mathrm{Yi}$ ) is the initial transverse (respectively, vertical) component of induction $\mathbf{B}$, and $\mathrm{B}_{\mathrm{ANH}} \mathrm{Xi}$ (respectively, $B_{\mathrm{ANH}} \mathrm{Yi}$ ) the transverse (respectively, vertical) component of the anhysteretic induction $\mathbf{B}_{\mathrm{ANH}}$ under inductor BOV measured previously on sensor $\mathrm{Si}$. $\xi$ has to be determined by fitting.

\section{E. Analytical Model for Longitudinal Inductor Field}

In the case of a longitudinal inductor field, given the physical symmetries of the problem, the measured transverse component $\mathrm{BXi}$ of induction is null for all sensors, given their location [see Fig. 1(a)].

An analytical solution to (7) cannot be found since measurements showed that the anhysteretic induction under a longitudinal polarization field depends on pressure. Nevertheless, variations of induction $\mathbf{B}$ with pressure can be separated into two terms, an exponential contribution in the low pressures area $(P<50$ bars $)$ as already found for the vertical inductor field, and an additive linear term in the high pressure area $(P>$ 70 bars) [see Fig. 4(b)]. Note that the laws (8) and (9) found for the vertical cases, are a particular case of the general law (10) and (11), with $b=0$.

For high levels of pressure, induction is close to its anhysteretic value, and consequently, follows the same behavior. For a longitudinal inductor field, variation of the anhysteretic induction is a linear function of pressure. Consequently, induction B can be approximated by a linear function for high pressure levels.

For lower pressure levels, the phenomenological behavior of induction is to tend towards its anhysteretic value. This behavior is driven by the first term of (7), and thus, is well approximated by an exponential function. Finally, approached analytical solutions to (7) for the vertical and longitudinal components BY and $\mathrm{BZ}$ of $\mathbf{B}$ are proposed

$$
B Y i(P, \boldsymbol{B} \mathbf{0} \boldsymbol{L})=a_{y}(\boldsymbol{B} \mathbf{0 L}) e^{-\frac{P^{2}}{2 E \xi}}+b_{y}(\boldsymbol{B} \mathbf{0 L}) P+c_{y}(\boldsymbol{B} \mathbf{0} \boldsymbol{L})
$$

$$
B Z i(P, \boldsymbol{B} \mathbf{0} \boldsymbol{L})=a_{z}(\boldsymbol{B} \mathbf{0} \boldsymbol{L}) e^{-\frac{P^{2}}{2 E \xi}}+b_{z}(\boldsymbol{B} \mathbf{0} \boldsymbol{L}) P+c_{z}(\boldsymbol{B} \mathbf{0} \boldsymbol{L})
$$

$\xi$ being intrinsic to the material, the value found previously for the case of a vertical inductor field is expected for (10).

\section{F. Physical Signification of $a, b$, and $c$ Parameters}

Determination of coefficients $a, b$, and $c$ can be done using a fitting algorithm, based on a HPP cycle measurement under $\mathbf{B 0 L}=40 \mu \mathrm{T}$, and $\mathbf{B 0 V}=80 \mu \mathrm{T}$, using (10), (11) as target functions. Nevertheless, a more physical approach can be used: for high pressure, $\mathrm{BYi}(P, \mathbf{B} \mathbf{L})$ and $\mathrm{BZi}(P, \mathbf{B} 0 \mathbf{L})$ are approximated by the linear terms of (10), (11). Consequently, using values found for $\mathbf{B}$ at $P=70$ bars and $P=100$ bars, determination of $b$ and $c$ can be achieved. In addition, $P=0$ bar in (10), (11) yields value for $a$. This approach was successfully tested, and the values found for $a, b$, and $c$ were close to the fitted values.

\section{G. Vectorial Analytical Law for Any Inductor Field}

Given the linearity of the measured induction $\mathbf{B}$ with regards to the inductor field (6), the predicted variation of induction on sensor B1 can be expressed for any inductor field $\mathbf{B} 0$ and a vectorial law for the variation of $\mathbf{B}$ with pressure can be expressed

$$
\begin{aligned}
\boldsymbol{B i}(\sigma, \boldsymbol{B 0})= & B X i(P, \mathbf{B O} \boldsymbol{V}) \boldsymbol{X}+B Y i(P, \boldsymbol{B} \mathbf{V}) \boldsymbol{Y} \\
& +B Y i(P, \boldsymbol{B} \mathbf{0} \boldsymbol{L}) \boldsymbol{Y}+B Z i(P, \boldsymbol{B} \mathbf{O} \boldsymbol{L}) \boldsymbol{Z} .
\end{aligned}
$$

\section{EXPERIMENTAL VALIDATION}

$\xi$ was determined using a fitting procedure with B0V $=$ $80 \mu \mathrm{T}$ [see Fig. 5(a)]. For $P$ expressed in bar, and $E=2.05 .10^{11} \mathrm{~Pa}, \xi \approx 2.5 .10^{-9} \mathrm{~J} / \mathrm{m}^{3}$. A subsequent HPP cycle under BOL $=40 \mu \mathrm{T}$ yielded expressions of (10) and (11). The value found in that case for $\xi$ using a fitting procedure was equal to the value found with $\mathbf{B 0 V}=80 \mu \mathrm{T}$, accounting for the expected isotropic magnetoelastic behavior of the material.

The model was then tested using several HPP cycles, under inductor fields BOL $+\mathbf{B O V}$, and several initial magnetic states. Results showed good agreement with measurements [see Fig. 5(b)]. The maximal relative error is less than $12 \%$.

\section{CONCLUSION}

A model for the variation of the external induction with internal pressure, measurable in the vicinity of our mockup, was presented. A general analytical law was derived for the variation of external induction components under a vertical and a longitudinal inductor field. Based on the linearity of external induction with regards to the inductor field, an analytical vectorial law was then proposed. Validity of this model was proved by comparison with a wide set of HPP cycles, performed under various inductor fields, and different initial magnetic states, with a relative error less than $12 \%$ with respect to measurements. Once this modeling of the external induction is achieved, further work has consisted in characterizing intrinsic magnetization $\mathbf{M}$ inside the shell: because of the non-negligible demagnetizing field, an inverse problem has to be solved and present results have been used to propose a dual vectorial model for magnetization variation due to stress [5].

\section{REFERENCES}

[1] D. C. Jiles, "Theory of the magnetomechanical effect," J. Phys. D: Appl. Phys., vol. 28, pp. 1537-1546, 1995

[2] L. Brugel and G. Rimet, "Interprétation des effets irréversibles des contraintes au moyen d'un modèle d'hystérésis dans l'espace," J. Phys. Radium, Paris, vol. 27, pp. 589-598.

[3] A. Viana, L.-L. Rouve, G. Cauffet, and J.-L. Coulomb, "Magneto-mechanical effects under low fields and high stresses-Application to a ferromagnetic cylinder under pressure in a vertical field," IEEE Trans. Magn., vol. 46, no. 8, pp. 2870-2875, Aug. 2010.

[4] O. Chadebec, J.-L. Coulomb, L.-L. Rouve, J.-P. Bongiraud, G. Cauffet, and P. Le Thiec, "Recent improvements for solving inverse magnetostatic problem applied to thin shell," IEEE Trans. Magn., vol. 38, no. 2, pp. 1005-1008, Feb. 2002.

[5] A. Viana, L.-L. Rouve, O. Chadebec, G. Cauffet, and J.-L. Coulomb, "Inverse problem approach to characterize and model magnetization changes in a thin shell structure undergoing magneto-mechanical effects," in Proc. CEFC, May 2010, vol. 47, no. 5. 